\title{
ANÁLISE ESPACIAL DE FRAGMENTOS FLORESTAIS NA BACIA DO RIO ITAPEMIRIM, ES ${ }^{1}$
}

\author{
Daiani Bernardo Pirovani², Aderbal Gomes da Silva ${ }^{3}$, Alexandre Rosa dos Santos ${ }^{4}$, Roberto Avelino \\ Cecílio $^{3}$, José Marinaldo Gleriani ${ }^{5}$ e Sebastião Venâncio Martins ${ }^{5}$
}

\begin{abstract}
RESUMO - Os objetivos deste trabalho foi mapear e analisar a estrutura da paisagem florestal em uma área representativa da Bacia do Rio Itapemirim, ES, por meio de índices de ecologia da paisagem. O mapeamento dos fragmentos florestais foi obtido utilizando técnicas de fotointerpretação na escala de 1:1500 do ortofotomosaico do ano 2007. Para o cálculo dos índices de ecologia, foi utilizada a extensão Patch Analyst dentro do aplicativo computacional ArcGIS 9.3. Foram encontrados 3.285 fragmentos florestais em toda a área, representando $17 \%$ de cobertura florestal. Os fragmentos mapeados foram divididos em classes de tamanho, sendo fragmentos pequenos aqueles menores que $5 \mathrm{ha}$; de tamanho médio aqueles entre 5 e 50 ha; e grandes os maiores que 50 ha. Os fragmentos pequenos encontravam-se em maior número (2.236), seguidos pelos fragmentos de tamanho médio (749) e, por último, pelos fragmentos grandes, que compreenderam apenas 100 manchas. O número de fragmentos de cada classe de tamanho possui relação inversa com a contribuição em área dessa classe dentro da fragmentação florestal. As análises quantitativas por meio de métricas da paisagem foram feitas com os grupos de índices de área; densidade e tamanho, forma; proximidade e área central, sendo este último obtido em diferentes simulações de efeito de borda (20,40, 60, 80, 100, 140 e 200 m). Para todos os índices houve diferenciações com relação às classes de tamanho dos fragmentos florestais. A maior parte dos fragmentos florestais da bacia, são pequenos, menores que 5 ha, sendo que a distância de borda de 100 metros elimina completamente a área central desses fragmentos. Apesar dos fragmentos grandes apresentarem os formatos mais irregulares, estes possuem maior índice de área central, mesmo sob o efeito da maior distância de efeito de borda.
\end{abstract}

Palavras-chave: Fragmentação florestal; Sistemas de Informações Geográficas; Índices de ecologia da paisagem.

\section{SPATIAL ANALYSIS OF FOREST FRAGMENTS IN THE ITAPEMIRIM RIVER BASIN, ES}

\begin{abstract}
The objectives of this study were to map and analyze the structure of forest landscape in a representative area of Itapemirim river basin, ES, through indexes of landscape ecology. The mapping of forest fragments was obtained using image interpretation techniques on a scale of 1:1500 of the ortho-image-mosaic of the year 2007. For the ecology metrics calculation, the extension Patch Analyst was used within the computational application ArcGIS 9.3. In general, 3285 forest fragments were found in all the area, representing $17 \%$ of the forest covering. The mapped fragments were divided into size classes, considering as small those fragments smaller than 5 ha, average fragments have size between 5 and 50 ha, and large fragments are larger than $50 \mathrm{ha}$. The small fragments were found in greater number $(2,236)$, followed by average fragments $(749)$, and finally the large fragments, comprising only 100 spots. The number of fragments of each size class has an inverse relationship with the contribution in area of the same class within the forest fragmentation. Quantitative characterizations through landscape metrics were made with groups of area index, density and size, shape, proximity and central area, which was obtained from different simulations of edge effect (20, 40, 60, 80, 100, 140 and $200 \mathrm{~m})$. For all the indexes there were differences on size classes of forest fragments. Most of the basin forest fragments
\end{abstract}

\footnotetext{
${ }^{1}$ Recebido em 22.11.2010 aceito para publicação em 27.02.2014.

${ }^{2}$ Instituto Federal de Educação, Ciência e Tecnologia do Espírito Santo, Campus Ibatiba, Ibatiba, Brasil. E-mail: <daiani.pirovani@ifes.edu.br>.

${ }^{3}$ Universidade Federal do Espírito Santo, Departamento de Ciências Florestais e da Madeira, Jerônimo Monteiro, Brasil. E-mail: <aderbalsilva@yahoo.com.br>e <racecilio@yahoo.com.br>.

${ }^{4}$ Universidade Federal do Espírito Santo, Departamento de Engenharia Rural, Alegre, Brasil. E-mail: <mundogeomatica@yahoo.com.br>.

${ }^{5}$ Universidade Federal de Viçosa, Departamento de Ciências Florestais, Viçosa, Brasil. E-mail: <gleriani@gmail.com>e

<venancioufv@gmail.com>.
} 
are small, less than $5 \mathrm{~h}$, and the distance of edge 100 meters completely eliminates the central area of these fragments. Although large fragments present the most irregular shapes, they have a higher index central area even under the effect of the greater distance of the edge effect.

Keywords: Forest Fragmentation; Geographic Information Systems; Indexes of Landscape Ecology.

\section{INTRODUÇÃO}

Segundo o Instituto Nacional de Pesquisas Espaciais - INPE (2009), restam atualmente apenas 7,91\% da área originalmente coberta pela Mata Atlântica. Esse ecossistema se torna cada vez mais frágil, despertando preocupações no desenvolvimento de estudos para manter a sustentabilidade dos remanescentes florestais. Assim, os fragmentos de diversos tamanhos e formas assumem fundamental importância para a perenidade do bioma Mata Atlântica.

A perda de ambientes naturais, aliado à fragmentação, tem resultado na formação de paisagens com pouca diversidade de hábitat (FAHRIG, 2003), com fragmentos isolados e de dimensões reduzidas (METZER, 2003). Um dos interesses em estudos ecológicos da paisagem, principalmente no que diz respeito à fragmentação florestal, está na quantidade e distribuição de determinado tipo de mancha ou classe (PEREIRA et al., 2001).

A riqueza e abundância de certas espécies florestais dependem das características estruturais dos fragmentos (METZGER, 2000). Para Murcia (1995), o efeito de borda está entre os principais fatores que afetam os padrões de diversidade e dinâmica das comunidades vegetais. Segundo Primack e Rodrigues (2001), o aumento na proporção de bordas eleva a temperatura do ar e o déficit de pressão do vapor, estendendo-se por muitos metros para o interior dos fragmentos. Esse processo promove um conjunto de mudanças no equilíbrio do ambiente, alterando as relações ecológicas entre populações.

Diversos estudos vêm sendo realizados visando conhecer a distribuição espacial de fragmentos florestais, a fim de se estabelecerem estratégias de conservação desses fragmentos. Trabalhos desta natureza foram realizados por Ferraz e Vettorazzi (2003) e por Batista et al. (2009), ambos no Estado de São Paulo; no Espírito Santo, Nascimento et al. (2006) mapearam os fragmentos florestais na Bacia do Rio Alegre; Paiva et al. (2010) realizaram análise dos fragmentos na delimitação de sítios florestais na Bacia do Rio Itapemirim; e Calegari et al (2010) analisaram a dinâmica dos fragmentos florestais no Município de Carandaí, em Minas Gerais.

Os objetivos deste trabalho foram mapear e analisar a estrutura da paisagem florestal em um trecho da Bacia do Rio Itapemirim, ES, por meio de índices de ecologia da paisagem. Procedeu-se à quantificação do arranjo espacial e à avaliação da complexidade estrutural dos fragmentos florestais, em termos de área, núcleo, forma e composição de borda; e da investigação das relações de conectividade entre fragmentos florestais.

\section{MATERIAL E MÉTODOS}

\subsection{Caracterização da área de estudo}

A Bacia Hidrográfica do Rio Itapemirim compreende 16 municípios localizados ao Sul do Estado do Espírito Santo e um município de Minas Gerais. O clima da região enquadra-se no tipo Cwa (inverno seco e verão chuvoso), de acordo com a classificação de Köppen. A região é caracterizada por uma topografia muito acidentada, intercalada por áreas planas.

A área correspondente a esse estudo se refere às interbacias codificadas com os números 77161, 77163 e 77165 do nível 5 do método de codificação proposto por Pfafstetter (1989), também denominado método das Ottobacias e que foi estabelecido pelo Conselho Nacional de Recursos Hídricos (CNRH) em sua Resolução $n^{\circ} 30$, para efeito de classificação e codificação das bacias hidrográficas em âmbito nacional. Essa área representativa da Bacia do Rio Itapemirim situa-se entre as coordenadas UTM 234.996,757 m e 311.931,093 m de longitude Oeste e 7.707.725,142 m e 7.685.540,420 $\mathrm{m}$ de latitude Sul. Possui uma área de $1.600 \mathrm{~km}^{2}$, abrangendo oito dos 17 municípios que compõem a referida bacia.

\subsection{Mapeamento dos fragmentos florestais}

O mapa dos fragmentos florestais foi obtido por meio da digitalização do ortofotomosaico de 2007, disponibilizado pelo Instituto Estadual de Meio Ambiente 
e Recursos Hídricos (IEMA), que possui escala de 1:35.000 e resolução espacial de $1 \mathrm{~m}$. A digitalização via tela foi feita na escala-padrão de 1:1.500 no aplicativo computacional ArcGIS 9.3 (ESRI, 2006), por meio de técnicas de fotointerpretação. Foi criado um arquivo vetorial poligonal para a classe fragmento florestal. A partir do mapa de fragmentação florestal da região de estudo, foi quantificada a área de cada fragmento de acordo com a tabela de atributos do próprio arquivo de imagem vetorial poligonal, tornando possível comparar os tamanhos dos diversos fragmentos florestais encontrados nesta parte da Bacia do Rio Itapemirim.

Os fragmentos na área foram relacionados à classe de tamanho aos quais pertencem e são classificados como pequenos, médios e grandes, definindo-se como pequenos os fragmentos com área menor que 5 ha, fragmentos médios aqueles com área entre 5 e 50 ha e fragmentos grandes aqueles com área maior que 50 ha.

\subsection{Análise dos fragmentos florestais por meio de métricas da paisagem}

A análise dos índices de ecologia da paisagem foi feita com base no mapa de fragmentos florestais gerado, sendo os valores obtidos pelo aplicativo computacional ArcGIS 9.3, através da extensão Patch Analyst. As métricas foram calculadas utilizando a versão para dados vetoriais (Patch.) para o arquivo de fragmentação florestal obtido na etapa anterior.

No sentido de evitar a utilização de métricas redundantes, optou-se por utilizar os seguintes grupos de métricas: índices de densidade de fragmentos; tamanho; forma dos fragmentos; índices de borda; área central; e o índice de proximidade entre os fragmentos, definidos de acordo com McGarigal e Marks (1995).

Para melhor comparação entre o grau de conservação e o tamanho dos fragmentos florestais mapeados neste estudo, foi realizada a análise dos índices de ecologia da paisagem para cada uma das classes de tamanho (pequena, média e grande) e para todos os fragmentos, sem distinção de tamanho, a fim de obter valores médios dos índices para todos os remanescentes da área. Por meio das métricas de tamanho, obtiveram-se a área total da classe fragmento florestal, o tamanho médio dos fragmentos, o desvio- padrão e coeficiente de variação. Com as métricas de densidade de fragmentos, foram obtidos o número de fragmentos em toda a área e a densidade de fragmentos a cada 100 ha da área de estudo.

As métricas de forma exigem a adoção de uma paisagem-padrão para efeito de comparação. A forma dos fragmentos foi comparada com o formato de um círculo para o cálculo da razão perímetro/área, visto que compara o valor desse índice a um círculo quando o arquivo é vetorial e a um quadrado para arquivos raster ou matricial. Os valores de índice de forma mais próximos de 1 são ligados a formatos mais regulares.

Para cálculo das métricas de área central dos fragmentos, utilizaram-se as distâncias de 20, 40, 60, 80, 100, 140 e $200 \mathrm{~m}$ de borda, obtendo diferentes cenários para análises.

\section{RESULTADOS}

O mapeamento possibilitou contabilizar 3.285 fragmentos florestais em toda a área representativa da Bacia do Rio Itapemirim. Esses fragmentos correspondem a uma área de 28.267,18 ha (ha) de remanescentes florestais. Isso significa que, da área total em estudo (160.000 ha), cerca de 17\% são fragmentos florestais (Figura1).

A classe dos fragmentos grandes representou um pequeno percentual (3,04\%), no número total de fragmentos florestais, com apenas 100 fragmentos. O

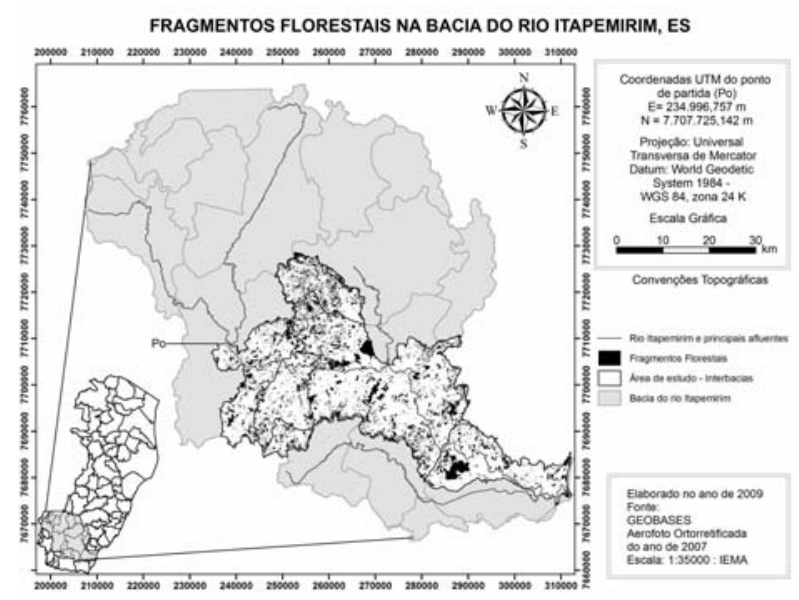

Figura 1 - Mapa dos fragmentos florestais em interbacias da Bacia do Rio Itapemirim, ES.

Figure 1 - Map of forest fragments in inter-basins of the Itapemirim river basin, ES.

Revista Árvore, Viçosa-MG, v.38, n.2, p.271-281, 2014 
total de fragmentos médios encontrados foi de 749 , correspondendo a $22,80 \%$ do total de fragmentos florestais de toda a área. Os fragmentos pequenos corresponderam à maioria em número (74,15\%), com 2.436 fragmentos. Os valores dos índices de ecologia da paisagem gerados por classes de tamanho e para todos os fragmentos da área encontram-se na Tabela 1. Os valores das métricas de área central, gerados para diferentes distâncias de faixa sob efeito de borda, são apresentados na Tabela 2 , a fim de investigar qual distância de borda exerce maior influência nos valores de área central dos fragmentos.

A análise da Figura 2 demonstra que, a partir do aumento da distância de borda para 40 m, os fragmentos pequenos sofreram redução drástica das métricas de área central, decaindo o valor do índice de área central total (TCAI) a 5,33\%. O TCAI mede a quantidade relativa de área central da classe considerada, e as espécies que ocupam a área central (interior do fragmento) não estão sujeitas aos mesmos impactos que aquelas inseridas na borda do fragmento sobre influência da matriz antrópica. Os fragmentos médios tiveram TCAI reduzido quase pela metade, passando a 35,33\% de área central em relação à área total da classe, enquanto os fragmentos grandes sofreram menor redução com o aumento da borda, indo a um valor de TCAI de 60,36\%. Assim, quanto menor o fragmento florestal, menor a quantidade relativa (percentual) de área central, ou seja, menor a área protegida de influência da matriz antrópica.

O tamanho médio das áreas centrais dos fragmentos é expresso pelo índice MCA (área central média). Os fragmentos grandes apresentaram valores de MCA muito superior às demais classes de tamanho, sendo esta a única métrica que apresentou crescimento com o aumento da distância de borda, fato ocorrido exclusivamente para a classe de fragmentos grandes (Figura 3). O aumento de MCA a partir da distância de borda de $100 \mathrm{~m}$ dos fragmentos grandes pode ser explicado pelo fato de que, à medida que a área central diminui continuamente de tamanho em consequência do aumento da faixa de efeito de borda, pode ocorrer que todo o fragmento seja constituído por hábitats com características de borda. Assim, quando é alta a porcentagem de área ocupada pelo maior fragmento da classe, ao computar a área central média, o total de área central dentro dessa classe de fragmentos passa a ser dividido por um número menor devido à redução do número de manchas que apresentam área sem influência do efeito de borda, expressa pelo número de áreas centrais (NCA).

Tabela 1 - Índices de ecologia da paisagem calculados pelo Patch Analyst para os fragmentos florestais da Bacia do Rio Itapemirim, ES.

Table 1 - Indexes of landscape ecology calculated by Patch Analyst for forest fragments in the Itapemirim river basin, ES.

\begin{tabular}{|c|c|c|c|c|c|c|}
\hline \multirow[t]{2}{*}{ Grupo } & \multirow[t]{2}{*}{ Índices } & \multirow[t]{2}{*}{ Unidade } & \multicolumn{4}{|c|}{ Classes de tamanho } \\
\hline & & & $\begin{array}{l}\text { Pequeno } \\
(<5 \text { ha })\end{array}$ & $\begin{array}{c}\text { Médio } \\
(5-50 \text { ha }) \\
\end{array}$ & $\begin{array}{c}\text { Grande } \\
\text { (> } 50 \text { ha })\end{array}$ & $\begin{array}{c}\text { Todos } \\
(0 \text { a }>50 \text { ha })\end{array}$ \\
\hline Área & $\mathrm{CA}$ & Hectares (ha) & $3.420,67$ & $11.309,50$ & $13.537,01$ & $28.267,18$ \\
\hline Densidade & MPS & Hectares (ha) & 1,40 & 15,10 & 135,37 & 8,61 \\
\hline \multirow[t]{3}{*}{ e tamanho } & NUMP & Adimensional & 2436 & 749 & 100 & 3.285 \\
\hline & PSSD & Hectares (ha) & 1,21 & 10,63 & 164,84 & 37,35 \\
\hline & PSCoV & Porcentagem & 85,54 & 70,25 & 122,79 & 433,85 \\
\hline \multirow[t]{2}{*}{ Borda } & $\mathrm{TE}$ & Metros (m) & $1.801 .330,95$ & $2.568 .015,5$ & $1.732 .072,3$ & $6.093 .977,15$ \\
\hline & ED & m/ha & 523,14 & 226,66 & 127,75 & 215,48 \\
\hline \multirow[t]{3}{*}{ Forma } & MSI & Adimensional & 1,85 & 2,50 & 4,29 & 2,07 \\
\hline & AWMSI & Adimensional & 1,99 & 2,75 & 4,81 & 3,65 \\
\hline & MPFD & Adimensional & 1,41 & 1,36 & 1,38 & 1,40 \\
\hline Proximidade & MNN & Metros (m) & 1.328 & 1.594 & 1.331 & 793,5 \\
\hline
\end{tabular}

* CA (Área de todas as manchas); MPS (Tamanho médio); NumP (Número de manchas); PScoV (Coeficiente de variação do tamanho); PSSD (Desvio-padrão do tamanho); MSI (Índice de forma médio); AWMSI (Índice de forma de área média ponderada); MPFD (Dimensão fractal da mancha média); TE (Total de bordas); ED (Densidade de borda); e MNN (Distância média do vizinho mais próximo).

* CA (Area of all patches); MPS (Medium Patch Size); NumP (Number of Patches); PScoV (Coefficient of variation of size); PSSD (Standard Deviation of the Size); MSI (Mean Shape Index); AWMSI (Area Weighted Mean Shape Index); MPFD (Mean Patch Fractal dimension ); TE (Total Edges); ED (Edge Density); e MNN (Mean distance from Nearest Neighbor).

Revista Árvore, Viçosa-MG, v.38, n.2, p.271-281, 2014 
Tabela 2 - Valores dos índices de ecologia da paisagem relativos às métricas de área central gerados pelo Patch Analyst utilizando diferentes distâncias de faixa sob efeito de borda, para as classes de tamanho dos fragmentos florestais na Bacia do Rio Itapemirim, ES.

Table 2 - Indexes values of landscape ecology regarding to the central area metrics generated by Patch Analyst using different distances of track under edge effect for the size classes of forest fragments in the Itapemirim river basin, ES.

\begin{tabular}{lcccccccc}
\hline \multicolumn{2}{c}{ Distância de borda } & 20 & 40 & 60 & 80 & 100 & 140 & 200 \\
\hline Pequeno (< 5 ha) & TCA & 921,81 & 183,25 & 183,25 & 23,44 & 1,25 & 0 & 0 \\
& TCAI & 26,79 & 5,33 & 5,33 & 0,68 & 0,04 & 0 & 0 \\
& CACoV & 132,56 & 107,65 & 107,65 & 89,79 & 59,16 & 0 & 0 \\
& CASD & 0,64 & 0,36 & 0,36 & 0,18 & 0,07 & 0 & 0 \\
& NCA & 1899 & 547 & 547 & 117 & 10 & 0 & 0 \\
& MCA & 0,49 & 0,34 & 0,34 & 0,20 & 0,12 & 0 & 0 \\
\hline Médio (5- 50 ha) & TCA & $6.860,2$ & $4.003,1$ & $4.003,1$ & $2.217,7$ & $1.170,5$ & 272,4 & 44,5 \\
& TCAI & 60,54 & 35,33 & 35,33 & 19,57 & 10,33 & 2,40 & 0,39 \\
& CACoV & 190,99 & 183,52 & 183,52 & 179,27 & 161,21 & 144,11 & 146,20 \\
& CASD & 7,23 & 5,26 & 5,26 & 3,88 & 3,02 & 1,75 & 1,10 \\
& NCA & 1.813 & 1.398 & 1.398 & 1.024 & 625 & 224 & 59 \\
& MCA & 3,78 & 2,86 & 2,86 & 2,17 & 1,87 & 1,22 & 0,75 \\
\hline Grande (> 50 ha) & TCA & $10.433,7$ & $8.188,3$ & $8.188,3$ & $6.532,5$ & $5.279,2$ & $3.568,3$ & $2.517,12$ \\
& TCAI & 76,92 & 60,36 & 60,36 & 48,16 & 38,92 & 26,30 & 18,56 \\
& CACoV & 50,38 & 49,41 & 49,41 & 47,92 & 48,09 & 45,73 & 44,33 \\
& CASD & 66,38 & 66,11 & 66,11 & 67,71 & 68,25 & 73,52 & 76,07 \\
& NCA & 792 & 612 & 612 & 463 & 372 & 222 & 145 \\
& MCA & 13,17 & 13,38 & 13,38 & 14,11 & 14,19 & 16,07 & 17,36 \\
\hline
\end{tabular}

*TCA (Área central total); TCAI (Índice de área central total); MCA (Área central média); NCA (Número de áreas centrais); CACoV (Coeficiente de variação de área central); e CASD (Desvio-padrão de área central).

*TCA (Total core area); TCAI (Total core area index); MCA (Mean core area); NCA (Number of Core Areas); CACoV (Central Area Coefficient of variation); e CASD (Central Area Standard deviation).

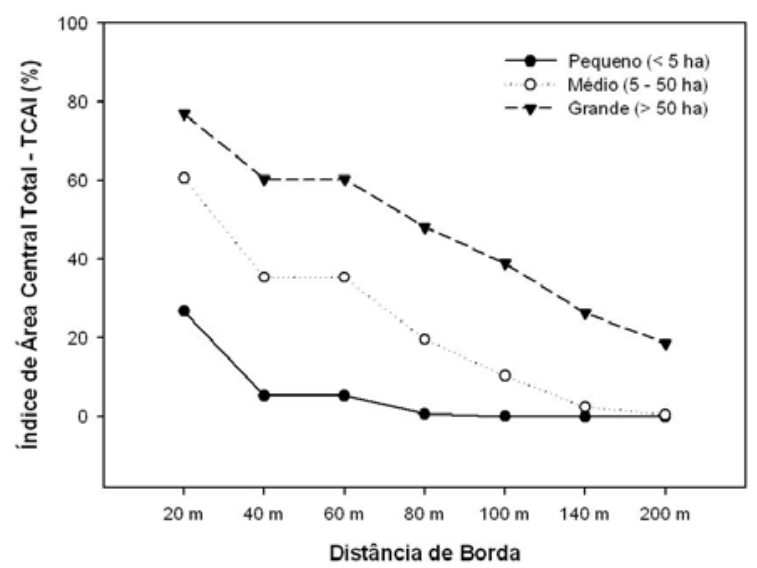

Figura 2 - Comportamento do índice de área central total com o aumento da distância de borda nas classes de tamanho dos fragmentos florestais.

Figure 2-Behavior of the total central area index with the distance increasing from edge to the size classes of forest fragments.

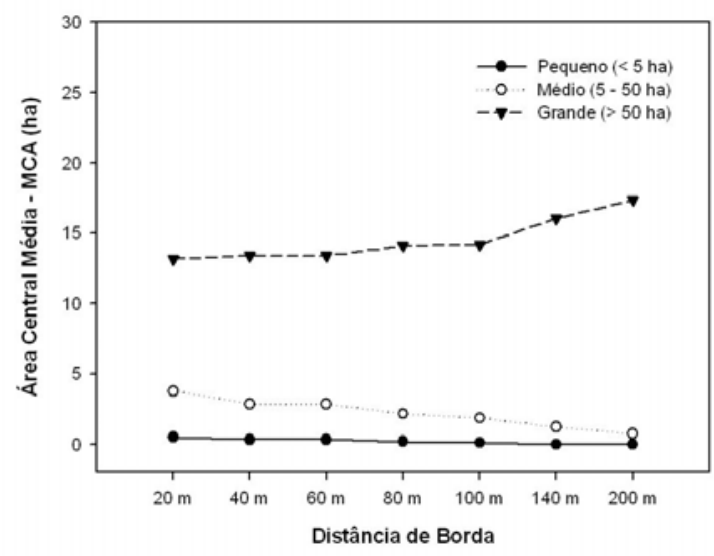

Figura 3 - Comportamento da área central média com o aumento da distância de borda nas classes de tamanho dos fragmentos florestais.

Figure 3-Behavior of the average central area with increasing distance from the edge in the size classes of forest fragments.

Revista Árvore, Viçosa-MG, v.38, n.2, p.271-281, 2014 


\section{DISCUSSÃO}

A área do fragmento é, em geral, o parâmetro mais importante para explicar as variações de riqueza de espécies. O mapa dos fragmentos florestais mostra que a maior parte dos remanescentes de Mata Atlântica nesta área da bacia do rio Itapemirim encontra-se em fragmentos pequenos (menor que $5 \mathrm{ha}$ ). Isso significa que essas áreas estão pouco conservadas, pois, de acordo com Metzger (1999), a riqueza diminui quando a área do fragmento fica menor do que as áreas mínimas necessárias para a sobrevivência das populações. A relação entre o número de fragmentos e área que eles ocupam é inversa, isto é, os fragmentos da classe de tamanho pequenos apresentaram maior número de manchas. No entanto, a soma de suas áreas representou o menor percentual da área total de fragmentos florestais mapeados, enquanto os fragmentos grandes, embora em menor número, apresentaram áreas significativas e foram responsáveis pela maior cobertura florestal em área. Tal condição é semelhante à observada por Almeida e Moro (2007), ao realizar a análise espacial dos fragmentos florestais no Parque Nacional dos Campos Gerais, onde constatou que a maior parte dos fragmentos florestais possuía área inferior a 5 ha, entretanto correspondiam a apenas 7,5\% da área total.

Diversos trabalhos apontam que a ocorrência de grande quantidade de pequenos fragmentos florestais é comum em paisagens de Floresta Atlântica. Cemin et al. (2009), analisando a composição e configuração da paisagem na subbacia do Arroio Jacaré, RS, constataram que $87,82 \%$ dos fragmentos florestais eram menores que 1 ha. Valente e Vettorazzi (2005), ao analisarem a estrutura da paisagem na Bacia do Rio Corumbataí, também obtiveram maior número de fragmentos pequenos (menores que 5 ha), correspondendo de 12 a 34\% da área total. A área em estudo encontra-se em um estado de fragmentação próximo ao observado por esses autores, pois o percentual em área dos fragmentos pequenos foi de 12,10\%, enquanto há alto número de fragmentos presentes nesta classe de tamanho (2.436), caracterizando um avançado processo de fragmentação florestal. O principal problema desse padrão é que os pequenos fragmentos estão mais sujeitos ao efeito de borda.

Segundo Forman e Godron (1986), os grandes fragmentos são importantes para a manutenção da biodiversidade e de processos ecológicos em larga escala. No entanto, os pequenos remanescentes também cumprem funções relevantes ao longo da paisagem, podendo funcionar como elementos de ligação, trampolins ecológicos (stepping stones) entre grandes áreas, promover aumento no nível de heterogeneidade da matriz e atuar como refúgio para espécies que requerem ambientes particulares que só ocorrem nessas áreas. Com base nisso, deve-se ressaltar que os fragmentos com pequenas áreas merecem atenção especial, sob o risco de serem extintos com o decorrer dos anos, caso não sejam adotadas propostas de manejo que promovam aumento de sua área e uma interligação com fragmentos próximos e maiores, pois remanescentes de área pequena apresentam frágeis padrões de sustentabilidade ao longo do tempo. Ressalta-se ainda que muitos fragmentos possam ser, na realidade, núcleos de colonização de florestas secundárias.

O índice de tamanho médio (MPS) representa a área média dos fragmentos da classe, nos fragmentos grandes. Esse índice apresentou o valor de 135,37 ha, porém, por meio do elevado valor do desvio-padrão do tamanho da mancha (PSSD = 164,84 ha), percebeu-se a alta variabilidade nos valores de área dos fragmentos grandes, mostrando que entre estes se podem encontrar de tamanhos mínimos para esse grupo (50 ha) até valores muito acima da média, como o do maior fragmento, que possui 1.306 ha. Alta variabilidade é comum em estudos de ecologia da paisagem, e Calegari et al. (2010) também observaram elevados coeficientes de variação em relação à área média dos fragmentos, indicando alta heterogeneidade espacial da paisagem. O MPS, analisando todas as classes de tamanho dos fragmentos conjuntamente, possui valor de 8,61 ha, no entanto o valor elevado do coeficiente de variação do tamanho da mancha (PSCoV $=433,85 \%$ ) indica alto desvio nos valores de área dos fragmentos florestais. Essa predominância de fragmentos pequenos na paisagem indica um histórico de fragmentação, no qual a cobertura florestal da região foi sendo retirada para dar lugar às áreas de pastagem, ficando os remanescentes florestais presentes apenas nos locais de mais difícil acesso e com área reduzida. Isso diminui consideravelmente a biodiversidade de espécies, visto que fragmentos de tamanho reduzido não apresentam as características ambientais suficientes para abrigar diversidade, e as diferentes espécies apresentam exigências distintas de luz, fertilidade, ventos, umidade e temperatura.

Quanto às métricas de borda, o total de borda (TE) representa a soma do perímetro de todos os fragmentos. O menor valor de TE foi obtido na classe dos fragmentos

Revista Árvore, Viçosa-MG, v.38, n.2, p.271-281, 2014

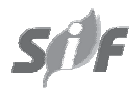


grandes $(1.732 .072 \mathrm{~m})$, já os fragmentos médios apresentaram o maior TE (2.568.015,5 m), e a classe de fragmentos pequenos apresentou valor intermediário em relação aos demais tamanhos (1.801.330,95 m). Os fragmentos menores apresentaram maior densidade de bordas (ED), com 523,14 m de borda por hectare, contra 226,66 m/ha dos fragmentos médios e 127,75 $\mathrm{m} /$ ha dos fragmentos grandes. Essa diferença na quantidade de bordas, quando se considera a densidade, é devida aos valores de área ocupados por cada classe de tamanho dos fragmentos, sendo a densidade de bordas inversamente proporcional à área ocupada por cada classe.

Esses resultados apontam para um menor efeito de borda nos fragmentos grandes, indicando maior grau de conservação, pois, de acordo com Rodrigues e Nascimento (2006), a borda é um limite permeável sujeito a impactos advindos da matriz exterior antrópica, que causa constante degradação. Tal fato acarreta alterações na composição de espécies e na estrutura da vegetação, devido à influência de vários processos e mudanças nos fatores abióticos e bióticos dos remanescentes, como alterações no microclima, aumento na temperatura do ar, aumento na intensidade dos ventos e diminuição na umidade do ar e do solo (KAPOS et al., 1997; SIQUEIRA et al., 2004). Isso, além do aumento da intensidade luminosa no interior dos fragmentos, em razão da abertura do dossel (KAPOS, 1989), essas alterações microclimáticas geram diversas mudanças na comunidade vegetal dos remanescentes, como aumento das taxas de mortalidade de árvores na proximidade da borda (LAURANCE et al., 2001), diminuição da riqueza e abundância de espécies lenhosas e herbáceas na proximidade da borda (FONTOURA et al., 2006), diminuição da densidade de plântulas em fragmentos e influência na dispersão de sementes (BARRERA et al., 2007).

O impacto do efeito de borda está relacionado à forma do fragmento, sendo maior a borda quanto mais irregular o formato, estando este diretamente ligado à relação entre o perímetro e a área; quanto menor essa relação, menor também será a borda e vice-versa, fragmentos de formatos mais regulares têm a razão borda área minimizada e, portanto, o centro da área está mais distante das bordas. Assim, a área central encontrase isolada dos fatores externos, enquanto fragmentos de formatos mais irregulares têm maior proporção de bordas, fazendo que ocorra maior interação com a matriz, o que, com o tempo, irá influenciar a qualidade da estrutura desses ecossistemas. O índice de forma (MSI) revelou que os fragmentos pequenos possuem formato mais regular (MSI $=1,85$ ) quando comparados com os fragmentos médios e grandes que apresentaram valores de MSI de 2,50 e 4,29, respectivamente; os valores do índice de forma médio ponderado pela área (AWMSI) foram superiores ao observado para o índice de forma médio (MSI), indicando que os fragmentos de maior área têm forma mais irregular que a média. Essa relação existe porque, para o cálculo do AWMSI, considera-se o tamanho do fragmento, no entanto se sabe que fragmentos pequenos são mais sujeitos ao efeito de borda do que os grandes.

O grau de isolamento dos fragmentos, expresso pela distância média do vizinho mais próximo (MNN), apresentou resultado semelhante nas três classes de tamanho consideradas (Tabela 1). Ao analisar a distância entre todos os fragmentos, sem distinção de classe de tamanho ( $\mathrm{MNN}=793,5 \mathrm{~m}$ ), o grau de isolamento decresce. Assim, é importante considerar os fragmentos de menor tamanho, como trampolins ecológicos dentro da paisagem. De maneira geral, pode-se considerar que os fragmentos dessa área representativa da Bacia do Rio Itapemirim possuem alto grau de isolamento, pois sua matriz antrópica é constituída, em sua maior parte, por pastagem, sendo baixa a permeabilidade entre este uso da terra e os fragmentos florestais, dificultando diversas interações ecológicas, tanto para a flora quanto para a fauna. Awade e Metzger (2008), por exemplo, observaram que algumas espécies de aves de subbosque evitam cruzar áreas abertas com distâncias superiores a $40 \mathrm{~m}$.

A área central de um fragmento de floresta é, segundo McGarigal e Marks (1995), melhor indicativo da qualidade dos fragmentos do que sua área total, sendo afetada diretamente pela forma e borda dos fragmentos. Dessa maneira, um fragmento pode ser largo o suficiente para sustentar algumas espécies, mas não conter área central suficiente e, sobretudo, manter a integridade de sua estrutura natural (TURNER; GARDNER, 1990).

Para uma borda mínima de $20 \mathrm{~m}$, a porcentagem de área dos fragmentos pequenos ocupada pelas áreas interiores, expressa pelo TCAI, foi de $26,79 \%$, e isso significa que $73,21 \%$ da área total dos fragmentos pequenos estão sob o efeito de borda de 20 m. O TCAI dos fragmentos médios foi de 60,54\%, representando menor efeito de borda do que nos fragmentos menores.

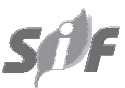

Revista Árvore, Viçosa-MG, v.38, n.2, p.271-281, 2014 
No entanto, observa-se que o valor de NCA (1.813) é maior que o número total de fragmentos médios (749), o que se deve ao fato de que alguns fragmentos, devido à irregularidade de seu formato, apresentaram mais de uma área central. Isso ocorre porque, em fragmentos com formatos recortados, a área de influência do efeito de borda pode chegar ao ponto de extremidades opostas sob efeito de borda se encontrarem, excluindo-se a área central nessa região do fragmento, ao passo que, no mesmo fragmento, extremidades mais distantes entre si podem computar duas áreas centrais distintas. Nesse cenário influenciado pela forma, o fragmento em questão passa a apresentar duas áreas centrais menores (protegendo menor número de espécies dos efeitos da matriz) em vez de uma área central maior, acontecendo o mesmo com os fragmentos grandes. O valor de NCA encontrado na borda de 20 m correspondeu a 792 áreas centrais, enquanto nessa classe de tamanho existiam, na área, apenas 100 fragmentos, demonstrado a influência do formato irregular dessa classe de tamanho (alto valor de MSI).

Em todas as classes de tamanho dos fragmentos florestais, o aumento da distância de borda de $40 \mathrm{~m}$ para 60 m não representou nenhuma mudança nos valores das métricas de área central, ou seja, esse acréscimo de 20 m na faixa de borda sobre influência das formas dos fragmentos não interferia nos impactos já advindos da matriz antrópica, mantendo o mesmo valor de área central. A simulação com a distância de borda de 80 m gerou redução mais drástica nas métricas de área central dos fragmentos pequenos, e os fragmentos médios e grandes sofreram menores reduções de área central para essa distância de borda, apresentando TCAI de 19,57\% e 48,16\%, respectivamente. Em estudo realizado por Cemin et al. (2009) em fragmentos florestais no Rio Grande do Sul, para uma distância de borda de $50 \mathrm{~m}$, os resultados indicaram que aproximadamente 40\% das matas ainda apresentavam área central com condições de suporte para fauna de pequeno e médio portes.

A distância de borda de 100 m levou os índices de área central dos fragmentos pequenos a resultados desprezíveis, sendo o TCAI de 0,04\%. Para as distâncias de borda de 140 m e 200 m, as métricas de área central nos fragmentos pequenos deixam de existir, e isso significa que, nessas condições, essa classe de tamanho se encontrava dominada pelo efeito de borda, estando todos os fragmentos suscetíveis à influência completa da matriz. Assim, considerando apenas a análise espacial e a influência do tamanho e forma do fragmento, independentemente das variáveis ambientais, a distância máxima alcançada pelo efeito de borda, sem extinguir a área central dos fragmentos, foi de $100 \mathrm{~m}$. E essa faixa é recomendada para trabalhos em nível espacial nos fragmentos dessa região, nos quais não fossem possíveis maiores detalhamentos no campo e sem considerar as alterações ambientais, pois, segundo Rodrigues e Nascimento (2006), uma borda recém-criada, em termos teóricos, pode ser estruturalmente homogênea ou muito semelhante ao interior florestal. E ao longo do tempo irão ocorrer outras transformações, relacionadas, em grande parte, aos efeitos de borda, que podem resultar uma comunidade mais heterogênea nesse limite.

Nos fragmentos grandes, o aumento na distância de borda levou a diminuições nas métricas de áreas centrais menores e mais gradativas. A área central total foi de $5.279,2$ ha, quando na largura de borda considerada de $100 \mathrm{~m}$, e apresentou valores de 3.568,3 ha e 2.517,12 ha, quando se considerou a faixa de borda de $140 \mathrm{~m}$ e $200 \mathrm{~m}$, respectivamente. A porcentagem de toda a área dessa classe livre de efeito de borda expressa pelo TCAI foi de 38,92\%, 26,30\% e 18,56\%, nas distâncias de 100, 140 e 200 m, respectivamente. Nota-se que nessa classe de tamanho a simulação com a maior distância de borda (200 m) não levou à quase ou total dominação do efeito de borda sobre os fragmentos, como ocorrido com os fragmentos médios e pequenos. A explicação para o aumento da área central média MCA no grupo dos fragmentos grandes está na presença de fragmentos muito superiores em área expressa pelo alto coeficiente de variação do tamanho da mancha (PSCoV = 433,85\%) para essa classe de tamanho e pela diminuição no valor do número de fragmentos com a área central (NCA). Isso fez que o valor médio aumentasse, pois o total de áreas centrais foi dividido por um número de fragmentos (NCA) menor.

Diversos trabalhos apontam para diferentes faixas de borda. Fontoura et al. (2006) propuseram um efeito de borda em torno de 50 m para Floresta Ombrófila Mista. Já Nascimento e Laurence (2006) encontraram maior biomassa e densidade de árvores e arvoretas de espécies pioneiras em fragmentos florestais com menos de 300 m de distância da borda do que em locais com mais de $300 \mathrm{~m}$ da borda. Na visão de Slaviero (2007), ao analisar a fragmentação florestal na região

Revista Árvore, Viçosa-MG, v.38, n.2, p.271-281, 2014

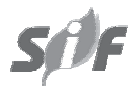


Norte do Rio Grande do Sul, a faixa de borda considerada foi de 100 m. Porém, Primack e Rodrigues (2001) relataram em Floresta Estacional Semidecidual alterações em déficit de pressão de vapor e em composição florística em largura de $35 \mathrm{~m}$ de borda. Contudo, percebeu-se que não havia padronização entre as distâncias de borda utilizadas nos estudos de fragmentos florestais, estando esta dependente dos objetivos do trabalho e do tipo de formação na qual o fragmento está inserido. Outro fator que pode influenciar é o gradiente topográfico, pois a influência do relevo pode intensificar ainda mais os impactos já existentes na borda, pois, quanto mais elevada a cota, mais forte a drenagem e, quanto mais forte o efeito de borda, mais os solos tendem a perder umidade (KAPOS et al., 1997; MURCIA, 1995). No trabalho de Souza et al. (2003), o efeito de borda potencializou o déficit hídrico presumido nas cotas mais altas e o incrementou nas cotas mais baixas. Assim, muitas espécies arbóreas mostraram clara preferência por dois hábitats: a baixa encosta, com solos mais úmidos e férteis; e a alta encosta, com solos menos úmidos e férteis e mais sujeita ao efeito de borda.

Neste estudo, considerando apenas a análise espacial com base no tamanho dos fragmentos, a distâncialimite de faixa sob efeito de borda para estimar a área central dos fragmentos florestais foi de $100 \mathrm{~m}$, pois valores acima deste eliminam por completo as áreas centrais dos fragmentos pequenos.

\section{CONCLUSÕES}

A maior parte dos fragmentos florestais nesta área representativa da bacia do rio Itapemirim, são fragmentos pequenos, menores que 5 ha, indicando um alto grau de fragmentação florestal nesta paisagem.

Quanto mais irregulares os formatos, maior foi o total de bordas dos fragmentos. Apesar dos fragmentos grandes apresentarem os formatos mais irregulares, estes possuem maior índice de área central, mesmo sob o efeito da maior distância de efeito de borda.

Para a realização de estudos de análise espacial com fragmentos florestais nesta região, ao se utilizar uma distância de borda a partir de 100 metros, se desconsidera por completo a área central dos fragmentos pequenos, pois estes estariam totalmente sob o efeito de borda.

\section{REFERÊNCIAS}

ALMEIDA, C. G.; MORO, R. S. Análise da cobertura florestal no Parque Nacional dos Campos Gerais, Paraná, como subsídio ao seu plano de manejo. Terr@ Plural, v.1, n.1, p.115-122, 2007.

AWADE, M.; METZGER, J. P. Importance of functional connectivity to evaluate the effect of habital fragmentation for three Atlantic Rainforest birds. Austral Ecology, n.33, p.863-871, 2008.

BARRERA, F. L. et al. Effects of varying Forest edge permeability on seed dispersal in a neotropical montane forest. Landscape Ecology, v.22, p.189-203, 2007.

BATISTA, E. R.; SANTOS, R. F.; SANTOS, M. A. Construção e análise de cenários de paisagem em área do Parque nacional da serra da Bocaina. Revista Árvore, v.33, n.6, p.1095-1108, 2009.

CALEGARI, L. et al. Análise da dinâmica de fragmentos florestais no município de Carandaí, MG, para fins de restauração florestal. Revista Árvore, v.34, n.5, p.871-880, 2010.

CEMIN, G.; PERICO, E.; REMPEL, C. Composição e configuração da paisagem da sub-bacia do Arroio jacaré, Vale do Taquari, RS, com ênfase nas áreas de florestas. Revista Árvore, v.33, n.4, p.705-711, 2009.

\section{ENVIRONMENTAL SYSTEMS RESEARCH} INSTITUTE (ESRI). ArcGIS Professional GIS for the desktop, versão 9.3, 2006.

FAHRIG, L. Effects of habitat fragmentation on biodiversity. Annual Review of Ecology, Evolution and Systematic, n.34, p.487-515. 2003.

FERRAZ, S. F. B.; VETTORAZZI, C. A. Identificação de áreas para recomposição florestal com base em princípios de ecologia de paisagem. Revista Árvore, v.27, n.4, p.575-583, 2003

FONTOURA, S. B.; GANADE, G.; LAROCCA, J. Changes in plant community diversity and composition across na edge between Araucaria Forest and pasture in South Brazil. Revista Brasileira de Botãnica, v.29, n.1, p.79-91, 2006.

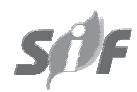

Revista Árvore, Viçosa-MG, v.38, n.2, p.271-281, 2014 
FORMAN, R. T. T.; GODRON, M. Landscape Ecology. New York: John Wilwy \& Sons, 1986. 619p.

INSTITUTO NACIONAL DE PESQUISAS ESPACIAIS - INPE. Ministério da Ciência e Tecnologia. Disponível em < http: //www.inpe.br> acesso em 24 ago. 2009.

KAPOS, V. Efffects of isolation on the water status of forest patches in the Brazilian Amazon.

Journal of Tropical Forest, v.5, n.2, p.173185, 1989.

KAPOS, V. et al. Edge related changes in environment and plant responses due to forest fragmentation in Central Amazonia. In: LAURANCE W. F.; BIERREGAARD R.O., (Ed.). Tropical forest remnants: Ecology, management, and conservation of fragmented communities New York: Chicago University Press, 1997. p.33-44.

LAURANCE, W. F. et al. Rain forest fragmentation and structure on Amazonian liana communities. Ecology, v,82, p.105-116, 2001.

MCGARIGAL, k.; MARKS, B. J. Fragstats: Spatial pattern analysis program for quantifying landscape structure. Reference manual. Corvallis: For. Sci. Dep. Oregon State University, 1995. 59p.

METZGER, J. P. Estrutura da paisagem e fragmentação: análise bibliográfica. Anais. Academia Brasileira de Ciências, v.71, n.3-I, p.445-463, 1999.

METZGER, J. P. Tree functional group richness and landscape structure in Brazilian tropical fragmented landscape. Ecological

Applications, v.10, n.4, p.1147-1161, 2000.

METZER, J. P. Estrutura da paisagem: o uso adequado de métricas. In: CULLEN JR.; L.; RUDRAN, R.; VALLADARES-PADUA, C. (Ed) Métodos de estudos em biologia da conservação e manejo da vida silvestre. Curitiba: Universidade Federal do Paramá, 2003. p.423-538.

MURCIA, C. Edge effects in fragmented Forest: implications for conservation. Tree, v.10, n.2, p.58-62, 1995.
NASCIMENTO, M. C. et al. Mapeamento dos fragmentos de vegetação florestal nativa da bacia hidrográfica do rio Alegre, a partir de imagens do satélite IKONOS II. Revista Árvore, v.30, n.30, p.389-398, 2006.

NASCIMENTO, H. E. M.; LAURANCE, W. F. Efeitos de área e de borda sobre a estrutura florestal em fragmentos de floresta de terra-firme após 13-17 anos de isolamento. Acta Amazonica, v.36, n.2, p.183-192, 2006.

PAIVA, Y. G. et al. Delimitação de sítios florestais e análise dos fragmentos pertencentes na bacia do rio Itapemirim. Idesia, v.28, n.1, p.17-22, 2010.

PEREIRA, J. L. G. et al. Métricas da paisagem na caracterização da evolução da Amazônia.

Geografia, v. 26, n. 1, p. 59-90, 2001.

PFAFSTETTER, O. Classificação de bacias hidrográficas: metodologia de codificação. Rio de Janeiro: Departamento Nacional de Obras de Saneamento, 1989. 19p.

PRIMACK, R.; RODRIGUES, E. Biologia da conservação. Londrina: 2001. 327p.

RODRIGUES, P. J. F. P.; NASCIMENTO, M. T. Fragmentação florestal: breves considerações teóricas sobre efeitos de borda. Rodriguésia, v.57, n.1, p.63-74, 2006.

SIQUEIRA, L. P. et al. Using the variances of microclimate variables to determine edge effects in small forest Atlantic Rain Forest fragments, South-Eastern Brazil. Ecotropica, v.10, n.1, p.59-64, 2004.

SLAVIERO, L. B. et al. Estrutura, configuração e fragmentação da Paisagem na região norte do rio grande do sul, Brasil. In: CONGRESSO DE ECOLOGIA DO BRASIL, 8., 2007, Caxambu. Anais... Caxambu, 2007. p. 1-2.

SOUZA, J. S. et al. Análise das variações florísticas e estruturais da comunidade arbórea de um fragmento de floresta semidecídua às margens do rio Capivari, Lavras-MG. Revista Árvore, v.27, n.2, p.185-206, 2003. 
Análise espacial de fragmentos florestais na bacia do...

TURNER, M. G.; GARDNER, R. H. Quantitative methods in landscape ecology: the analysis and interpretation of landscape heterogeneity. New York: Springer-Verlag, 1990. 536 p.
VALENTE, R. O. A.; VETTORAZZI, C. A. Análise da estrutura da paisagem na Bacia do Rio Corumbataí. Scientia Florestalis, n.62, p.114119. 2005. 
